\title{
A SUBSTITUTE FOR THE PICONE FORMULA
}

\section{WALTER LEIGHTON}

It has been known for a number of years that the calculus of variations affords a powerful tool for the study of the oscillation of solutions of self-adjoint differential systems. ${ }^{1}$ It is the purpose of the present paper to demonstrate how it can be used to replace and extend the Picone theorem. ${ }^{2}$

Consider the pair of self-adjoint differential equations

$$
\begin{gathered}
\frac{d}{d x}\left(r u^{\prime}\right)+p u=0, \\
\frac{d}{d x}\left(r_{1} u^{\prime}\right)+p_{1} u=0,
\end{gathered}
$$

where, for definiteness, it is assumed that $r(x), r_{1}(x), r^{\prime}(x), r_{1}^{\prime}(x)$, $p(x), p_{1}(x)$ are continuous with $r$ and $r_{1}$ positive on the interval $a \leqq x \leqq b$. With these equations we associate the functional identity

$$
\begin{aligned}
\int_{a}^{b}[(r & \left.\left.-r_{1}\right) u^{\prime 2}+\left(p_{1}-p\right) u^{2}\right] d x \\
& =\left.r u u^{\prime}\right|_{a} ^{b}-\int_{a}^{b} u\left[\left(r u^{\prime}\right)^{\prime}+p u\right] d x-\int_{a}^{b}\left[r_{1} u^{\prime 2}-p_{1} u^{2}\right] d x .
\end{aligned}
$$

The proof of (2) requires simply the integration by parts of the term $r u^{\prime 2}$ in its left-hand member. In what follows it is convenient to admit to our discussion functions ${ }^{3} u(x)$ of class $C^{\prime}$ on the interval $a \leqq x \leqq b$ which vanish at $a$ and $b$.

Repeated use is made of the following well known result. ${ }^{4}$

LEMмA. If there exists an admissible curve $y=y(x)$ along which

$$
J=\int_{a}^{b}\left(R y^{\prime 2}-P y^{2}\right) d x<0
$$
1948.

Prescnted to the Society, September 10, 1948; received by the editors April 15,

${ }^{1}$ See, for example, Morse [2, Chap. IV]. Numbers in brackets refer to the bibliography at the end of the paper.

${ }^{2}$ Cf. Bôcher [1, p. 54], Ince [1, p. 225].

3 A function is said to be of class $C^{\prime}$ on an interval $a \leqq x \leqq b$ if it is continuous and has a continuous derivative on $a \leqq x \leqq b$.

${ }^{4}$ Cf. Morse [2, chap. 2]. 
a solution $\bar{y}(x) \not \equiv 0$ of the differential system

$$
\begin{aligned}
\left(R y^{\prime}\right)^{\prime}+P y & =0, \\
y(a) & =0
\end{aligned}
$$

will have a zero on the interval $a<x<b$.

We return now to equations (1.1) and (1.2) and suppose that $u(x) \neq 0$ is a solution of (1.1) having $x_{1}$ and $x_{2}$ as consecutive zeros $\left(x_{1}<x_{2}\right)$. We seek sufficient conditions that every solution $u_{1}(x)$ of $(1.2)$ shall have at least one zero on the interval $x_{1}<x<x_{2}$. To that end we evaluate (2) with $u, a, b$ replaced respectively by $u(x), x_{1}, x_{2}$, obtaining the fundamental relationship

$$
\begin{aligned}
\int_{x_{1}}^{x_{2}}\left[\left(r_{1} u^{\prime 2}(x)-p_{1} u^{2}(x)\right] d x\right. & \\
& =-\int_{x_{1}}^{x_{2}}\left[\left(r-r_{1}\right) u^{\prime 2}(x)+\left(p_{1}-p\right) u^{2}(x)\right] d x
\end{aligned}
$$

which for simplicity we write as $I_{1}=-I$. It is clear that if we prescribe conditions which will insure that $I$ is positive, $I_{1}$ will then be negative. It will follow from the lemma that every solution $u_{1}(x)$ of (1.2) which vanishes at $x_{1}$ will vanish again on the interval $\left(x_{1}<x<x_{2}\right)$. The elementary Sturm separation theorem then insures that every solution of (1.2) will then vanish at least once on the interval $x_{1}<x<x_{2}$.

The Sturm-Picone (SP) conditions provide that $r \geqq r_{1}$ and $p_{1} \geqq p$, with the latter inequality holding in the strict sense in at least one point of the interval of integration. Clearly these conditions will insure that $I>0$, and hence that all solutions of (1.2) will have a zero on $x_{1}<x<x_{2}$.

The SP conditions are clearly not very delicate. One does not need to require that the coefficients of $u^{\prime 2}$ and $u^{2}$ be essentially positive to insure that $I$ is positive. Indeed, we may apply the Lemma to $I$. We obtain at once the following result.

TheOREM. ${ }^{5}$ In equations (1.1) and (1.2) let $r>r_{1}$ and suppose that a solution $u(x)$ of (1.1) has $x=x_{1}$ and $x=x_{2}$ as consecutive zeros. If $p(x)$ and $p_{1}(x)$ are such that a solution $w(x) \neq 0$ of the differential system

$$
\frac{d}{d x}\left[\left(r-r_{1}\right) w^{\prime}\right]+\left(p-p_{1}\right) w=0,
$$

S It should be pointed out that this result can be derived as a consequence of a theorem due to Morse [2, p. 102]. 


$$
w\left(x_{1}\right)=0
$$

has the property that $w(x) \neq 0$ on the interval $x_{1}<x \leqq x_{2}$, every solution of (1.2) has at least one zero on the interval $x_{1}<x<x_{2}$. The conclusion of this theorem will remain valid if $w(x) \neq 0$ on $x_{1}<x<x_{2}$ and $w\left(x_{2}\right)=0$, provided the solution $u(x)$ does not satisfy equation (6.1). ${ }^{6}$

The condition $r>r_{1}$ which is desirable in order that the functional $I$ shall be nonsingular may appear at first glance to be unduly restrictive. It is easily seen however that the theorem permits the study of the oscillation of solutions of (1.1) and (1.2) even when $r \leqq r_{1}$. We may simply replace $r(x)$ and $p(x)$ by $k r(x)$ and $k p(x)$, respectively, where $k$ is any positive constant such that $k r(x)>r_{1}(x)$. Such a determination of $k$ is possible since $r(x)$ is positive throughout its domain of definition.

The observation above suggests incidentally the following simple extension of the SP theorem.

Corollary. Let $u(x) \neq 0$ be a solution of (1.1) which has zeros at $x=x_{1}$ and $x=x_{2}$. If there exists a positive constant $k$ such that both the conditions

$$
k r-r_{1} \geqq 0, \quad p_{1}-k p \geqq 0
$$

hold, every solution $u_{1}(x)$ of (1.2) has a zero on the interval $x_{1}<x<x_{2}$, provided that at some point of this interval the latter inequality holds in the strict sense.

The following example demonstrates that the theorem includes cases not covered by the SP theory even in the extended form suggested by the corollary.

EXAMPLE. The Legendre equation

$$
\frac{d}{d x}\left[\left(1-x^{2}\right) y^{\prime}\right]+12 y=0
$$

has the solution $y=P_{3}(x)=\left(5 x^{3}-3 x\right) / 2$ which vanishes at $x=0$ and at $x=(3 / 5)^{1 / 2}$. We shall show that every solution of the differential equation

$$
\frac{d}{d x}\left[\left(3 / 4-x^{2}\right) y^{\prime}\right]+8 y=0
$$

'A complete bibliography of oscillation and comparison theorems for SturmLiouville systems cannot be given here. Closely related papers are Bliss and Schoenberg [1] and Morse [1] to which the reader's attention is directed. 
has a zero on the interval $0<x<(3 / 5)^{1 / 2}$. Here the analogues of equations (6.1) and (6.2) are

$$
\frac{d}{d x}\left(w^{\prime} / 4\right)+4 w=0, \quad w(0)=0 .
$$

It is clear that $w(x)$ may be taken as $\sin 4 x$ which is positive on the interval $0<x<(3 / 5)^{1 / 2}$. An appeal to the theorem completes the proof. It is readily verified that the SP theory does not apply even in the extended form suggested by the corollary.

\section{Bibliography}

G. A. Bliss and I. J. Schomnderg

1. On separation, comparison and oscillation theorems for self-adjoint systems of linear second order differential equations, Amer. J. Math. vol. 53 (1931) pp. $781-$ 800 .

MAXIME BôcHer

1. Leçons sur les methodes de Sturm, Paris, Gauthier-Villars, 1917.

E. L. INCE

1. Ordinary differential equations, London, Longmans, 1927. MARSTON MORSE:

1. A generalization of the Sturm separalion and comparison theorems in $n$-space, Math. Ann. vol. 103 (1930) pp. 52-69.

2. The calculus of variations in the large, Amer. Math. Soc. Colloquium Publications, vol. 18, New York, 1934.

WASIIINGTON UNIVERSITY 